Int. J. Dev. Biol. 57: 375-381 (2013)

doi: $10.1387 / \mathrm{ijdb} .130046 \mathrm{jw}$

\title{
The Dictyostelium prestalk inducer DIF-1 directs phosphorylation of a bZIP transcription factor
}

\author{
YOKO YAMADA, YUZURU KUBOHARA ${ }^{1}$, HARUHISA KIKUCHI ${ }^{2}$, YOSHITERU OSHIMA ${ }^{2}$, \\ HONG-YU WANG, SUSAN ROSS and JEFFREY G. WILLIAMS* \\ ${ }^{1}$ College of Life Sciences, Welcome Trust Biocentre, University of Dundee, UK, ${ }^{2}$ Department of Molecular and Cellular \\ Biology, Institute for Molecular and Cellular Regulation, Gunma University, Japan and ${ }^{3}$ Laboratory of Natural Product \\ Chemistry, Tohoku University Graduate School of Pharmaceutical Sciences, Aoba-yama, Aoba-ku, Sendai, Japan
}

\begin{abstract}
DIF-1, a chlorinated hexaphenone produced by developing Dictyostelium cells, induces prestalk differentiation. DimB is a bZIP transcription factor that accumulates in the nucleus upon exposure to DIF-1, where it directly activates transcription of DIF-responsive genes. The signaling steps upstream of DimB and downstream of DIF-1 are entirely unknown. Analysis by mass spectrometry shows that incubation with DIF-1 rapidly stimulates phosphorylation at several sites in DimB. We characterize the most highly responsive site, S590, which is located very close to the C terminus. A point mutation in this site, S590A, does not inhibit DimB nuclear accumulation in response to DIF. However, this seems likely to reflect functional redundancy with other sites; because a panel of chemical variants on the structure of DIF-1 show a correlation between their potencies as inducers of DimB nuclear accumulation and their potencies as inducers of phosphorylation at S590. Furthermore, the S590A mutant is fully active in mutant rescue of a dimB null strain, arguing against an alternative role in transcriptional activation of target genes. We conclude that i) DIF-1 directs phosphorylation at S590, ii) although it is not essential for nuclear accumulation in response to DIF-1 correlative evidence, based upon a panel of DIF-1 related molecules, suggests that this modification may play a redundant role in the process. iii) We also present evidence that the kinase activity, which phosphorylates S590, is non-nuclear and that this signalling pathway is, in part at least, independent of the DIF-regulated STATc activation pathway.
\end{abstract}

KEY WORDS: Dictyostelium, DimB: DIF-1, activation, kinase

\section{Introduction}

Pattern formation in Dictyostelium occurs by the morphogenetic movements of partially differentiated but uncommitted precursor cells (reviewed by Gaudet et al., 2008; Kay and Thompson, 2009). Primary differentiation is either into prestalk cells, the direct precursors of the stalk and the basal disc, or into prespore cells. Extracellular cAMP signalling induces prespore differentiation while DIF-1 signalling induces formation of a subset of prestalk cells, the pstB cells, that are precursors of the basal disc (Keller et al., 2008; Saito et al., 2008; Yamada et al., 2011). DIF-1, a polyketide, is also necessary for correct gene expression in the pstO region, a band of cells that comprise the rear half of the prestalk zone (Thompson et al., 2000). Two genes that encode related extracellular matrix proteins, ecmA and ecmB, act as markers for these prestalk cell sub-types. A cap-site distal region of the ecmA promoter is selectively active in pstO cells, while pstB cells selectively express ecm $B$ (Early et al., 1993; Jermyn and Williams, 1996). Both promoters contain essential binding sites for DimB; a bZIP transcription factor that translocates to the nucleus and binds to the promoter when cells are exposed to DIF-1 (Huang et al., 2006; Yamada et al., 2011; Zhukovskaya et al., 2006).

DIF-1 is a chlorinated hexaphenone that is synthesised by the prespore cells (Kay and Thompson, 2001). The structural requirements for its biological activity are partially understood (Gokan et al., 2005) but the mechanism whereby it activates DimB is unknown. There is another DIF-1 regulated signaling pathway, that functions

Abbreviations used in this paper: DIF, differentiation inducing factor; STAT, Signal Transducer and Activator of Transcription.

\footnotetext{
*Address correspondence to: Jeffrey Williams. College of Life Sciences, WelcomeTrust Biocentre, University of Dundee, Dow St., Dundee, DD1 5EH, UK. Tel: +44-1382-385-823. Fax: +44-1382-344-211. E-mail: j.g.williams@dundee.ac.uk
} 


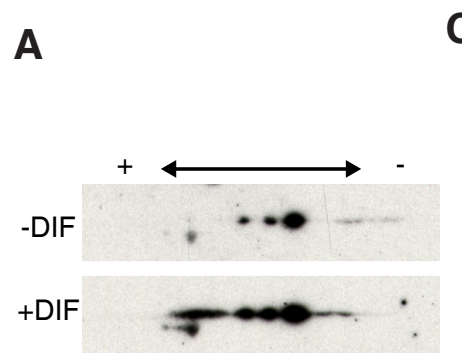

B

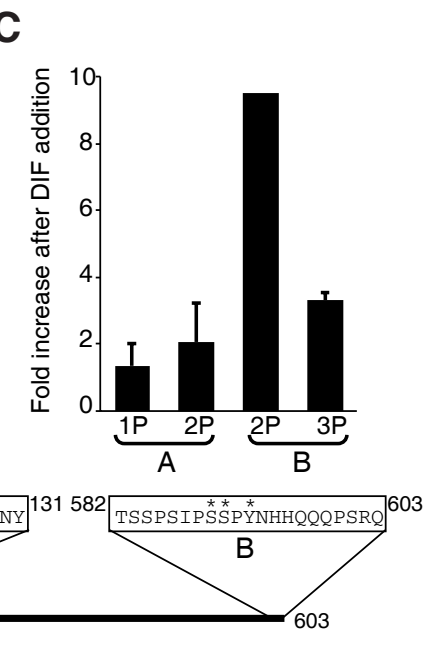

via a Signal Transducer and Activator of Transcription (STAT), and it is better characterised. Here phosphorylation of a tyrosine residue in STATc is regulated, by DIF-mediated inhibition of the PTP3 protein tyrosine phosphatase (Araki et al., 2008; Araki et al., 2010). In other eukaryotic organisms bZIP proteins are directly regulated by specific phosphorylation events (e.g. CREB and C/ EBP, respectively reviewed in Sharma et al., 2007 and Tsukada et al., 2011). Here we employ mass-spectrometry to analyse changes in DimB phosphorylation induced by exposure to DIF1. This identifies a strongly regulated site and we characterise it using a phospho-specific antibody.

\section{Results}

\section{DIF-1 induces phosphorylation of DimB}

Potential phosphorylation of DimB in response to DIF-1 was first investigated by performing 2D gel electrophoresis Western transfer using an antibody directed against DimB. Prior to the addition of DIF-1 there is a major spot and two satellite spots, of similar mobility to the major spot but with more acidic PIs (Fig 1A). After exposure to DIF-1 for 15 minutes the intensity of the two satellite spots increases significantly and an additional smear is observed at an even more acidic PI. These changes suggest a DIF-induced increase in phosphorylation, over a background level, and encouraged us to perform parent-ion scanning mass spectrometry to identify sites of modification.

The DimB substrate used for parent ionscanning was over-expressed in $\operatorname{dimB}$ null (dimB-) cells from its own promoter; by transforming cells with construct DimB:wt, which was inserted in an aminoglycoside resistance vector and selected at a high G418 concentration to achieve a high copy number. DimB was immuno-purified from untreated cells or from cells exposed to DIF-1 for $15 \mathrm{~min}$. When the parent-ion scans of the DIF-treated and untreated samples were overlaid, species that increase or decrease in apparent relative abundance could be identified. Identification of the phosphorylated peptides, that show a
Fig. 1. Identification of DIF-induced phosphorylations of DimB. (A) $2 D$ gel analysis Ax2 control cells, or cells treated with DIF-1 for 15 min, were lysed and analysed by 2D Western blotting using an anti-DimB antibody. (B) Sequences of two regions that change in phosphorylation level in response to DIF-1 Quantitation of phosphopeptides observed in pre79 scanning and subsequent MS/MS sequencing identified two peptide regions, $A$ and $B$, with increased levels of phosphorylation in DIF-treated cells. MS-MS for the 2P form of peptide B identified S589 and S590 as major sites of phosphorylation, although smaller peaks corresponding to fragments that contain pY592 were also observed. The positions of regions $A$ and $B$ relative to the bZIP domain of DimB are indicated and the predicted phosphorylation sites are asterisked. (C) Quantitation of the phosphorylation changes by mass spectrometry In this figure the predicted peptide amount in the DIF-induced peak relative to the same peak in the control sample is shown; expressed as an average +/-SD for peptides bearing the indicated degree of phosphorylation ( $n=2$ for the $2 P$ form of peptide $B$, and $n=3$ for the others). For $A$, the amounts of two peptides with the same degree of phosphorylation, but of slightly different length due to a mis-cleavage (indicated by a / in 1B), are summed.

quantitative change in response to DIF-1, could be made for only two regions: $A$ and $B$. Peptide $A 1$, and its slightly longer, incompletely cleaved form $\mathrm{A} 2$, are at the $\mathrm{N}$-terminus proximal boundary of the bZIP domain while peptide $B$ is located at the extreme $C$ terminus of DimB (Fig. 1B). There may well be other sites of regulated phosphorylation; because, as explained in Methods, some DIF-1 regulated peaks were unassigned and only just over half of the length of the protein can be accessed using this technique.

Regions A and B both contain three predicted sites of phosphorylation that are represented with different degrees of phosphorylation; there are $1 \mathrm{P}$ and $2 \mathrm{P}$ forms for peptides $\mathrm{A} 1$ and $\mathrm{A} 2$ while there are $2 \mathrm{P}$ and $3 \mathrm{P}$ forms for peptide $\mathrm{B}$. The stimulation of phosphorylation by DIF-1 in both regions was quantitated from multiple such parent ion scanning analyses. The average stimulation is slightly less than 2-fold for the combined $2 \mathrm{P}$ forms of peptide $A$ but is almost ten fold for the $2 \mathrm{P}$ form of peptide $\mathrm{B}$ (Fig. 1C). Because the latter induction is so much stronger, subsequent work was primarily directed to region $B$.

\section{Confirmation of DIF-1 regulated phosphorylation at S590}

The analysis of peptide B implicated two serine residues, S589 and S590, and a tyrosine residue, Y592, as sites of phosphorylation (Fig 1B). However, the Ms-Ms data suggested that the majority
A

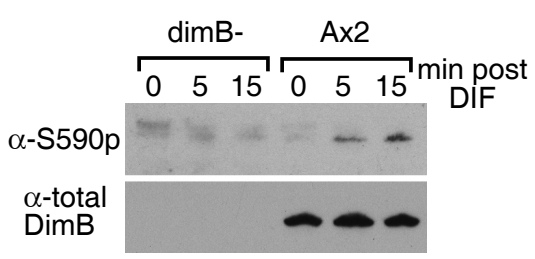

B
Fig. 2. Analysis of DIF-induced phosphorylation of S590 of DimB using a phospho-specific antibody. (A) Analysis of non-transformant strains ParentalAx2 cells and dimB-cells were treated with either DIF-1 (+) or $0.1 \%$ ethanol (-), as vehicle control, for the indicated times. After lysis and gel electrophoresis, the S590 phosphorylated form of DimB was detected by Western blotting using the phospho-specific antibody. (B) Analysis of transformant strains Cells derived from the $\operatorname{dimB}$ - strain, and over-expressing the wild type or the indicated point-mutated form of DimB, were treated with either DIF-1 or $0.1 \%$ ethanol for the indicated times. The 5590 phosphorylated form of DimB was detected as in Fig. 2 A. 


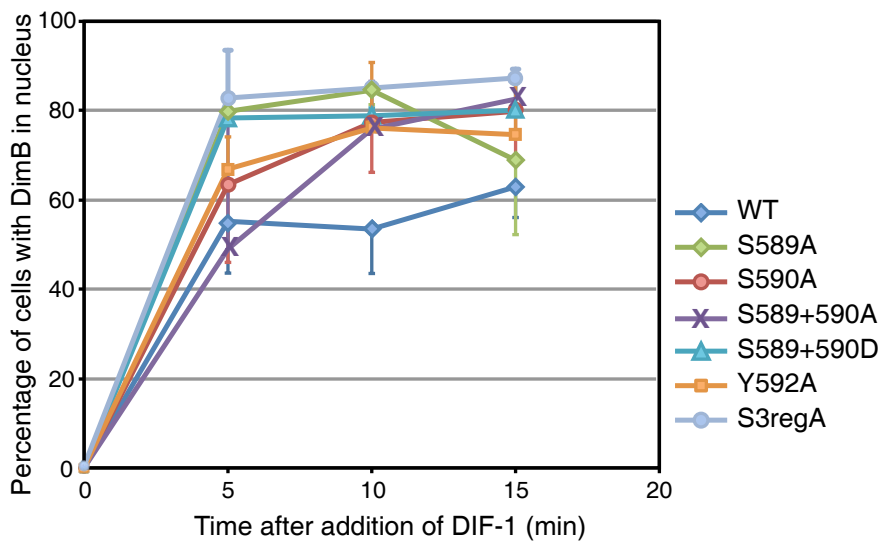

T S S P S I P S S P Y N H H Q Q P S R Q

T S S P I P A S Y N H H Q Q P S R - S589A

T S S P I P S P Y N H H Q Q P S Q - S590A

T S S P I P a A P Y H H Q Q P S R - S589+590A

T S S P S P d d P Y N H Q Q P S R - S589+590D

T S S P I P S P a NHH Q Q P S Q - Y592A

of phosphorylation was on the serine residues so Y592 was not further investigated. In order to confirm phosphorylation at sites S589 and S590, we attempted to raise phospho-specific antibodies against them. Antisera were prepared against each of the two uniquely phosphorylated peptides and purified by sequential chromatography on the phosphorylated and unphosphorylated forms of the respective peptide immunogens. Only the antibody against pS590 proved to be active. The antibody detects DimB in a Western transfer. Binding increases upon exposure to DIF-1 and binding at the position of DimB is not detected in extracts from dimB- cells (Fig. 2A).

Although it is reproducible the induced phosphorylation signal
Fig. 3. Nuclear accumulation in response to DIF-1 of DimB with point mutations in the regulated sites. DimB-derived cells, overexpressing wild type or mutated forms of DimB as indicated, were treated with DIF-1 and harvested at 5, 10 and 15 minutes. After fixing and staining with the total DimB antibody, the proportion of nuclei containing DimB was determined. The average value and $S D$ are shown $(n=3)$.

for the endogenous DimB protein is weak and there is another, smeared band at higher apparent molecular weight. Hence, as a further control for the specificity of the S590 phospho-specific antibody, we compared dimB- cells with the same cells transformed with DimB:wt (Fig. 2B). The latter cells show a much stronger induced signal: consistent with DimB being expressed from multiple copies and with the S590 antibody being specific. The specificity of the antibody was also assessed using cells transformed with a construct, DimB:S590A, bearing mutations that convert S590 into alanine. There is no detectable antibody binding to the DimB:S590A protein (Fig 2B). However, a construct in which the adjacent residue, S589, is mutated to alanine, in DimB:S589A, displays a DIF-regulated signal. In addition to further validating the specificity of the antibody, the latter result indicates that S589 does not act as a priming site for phosphorylation by the $\mathrm{S} 590$ kinase.

\section{Mutation of the S590 phosphorylation site does not affect DIF -induced nuclear translocation of DimB}

A series of point mutations, located in region $B$ of DimB, were created within the framework of the DimB:wt construct. The single mutants changed S589, S590 or Y592 into alanine. In one double mutant both S589 and S590 were changed into alanine. Another mutant changed the same two residues into, phospho-mimetic,
A

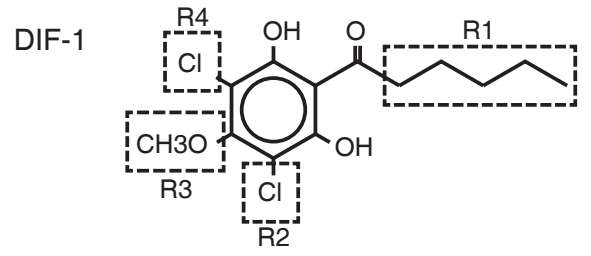

\begin{tabular}{lllll}
\hline & $\mathrm{R} 1$ & $\mathrm{R} 2$ & $\mathrm{R} 3$ & $\mathrm{R} 4$ \\
\hline DIF-1 & $(\mathrm{CH} 2) 4-\mathrm{CH} 3$ & $\mathrm{Cl}$ & $\mathrm{OCH} 3$ & $\mathrm{Cl}$ \\
DIF-1 $(+1)$ & $(\mathrm{CH} 2) 5-\mathrm{CH} 3$ & & & \\
DIF-1(+2) & $(\mathrm{CH} 2) 6-\mathrm{CH} 3$ & & & \\
DIF-1(3M) & $\mathrm{CH} 2-\mathrm{C}(\mathrm{CH} 3) 3$ & & & \\
DMPH & & $\mathrm{H}$ & & $\mathrm{H}$ \\
TH-DIF-1 & & & $\mathrm{OH}$ & \\
DIF-3 & & $\mathrm{H}$ & & \\
DIF-3(-1) & $(\mathrm{CH} 2) 3-\mathrm{CH} 3$ & $\mathrm{H}$ & & \\
DIF-3(+2) & $(\mathrm{CH} 2) 6-\mathrm{CH} 3$ & $\mathrm{H}$ & & \\
\hline
\end{tabular}

Fig. 4. Pharmacological analysis of the phosphorylation and nuclear translocation of DimB using DIF-1 related molecules as inducers. (A) A representation of the structure of DIF-1 and of related molecules Modifications at the positions indicated by the

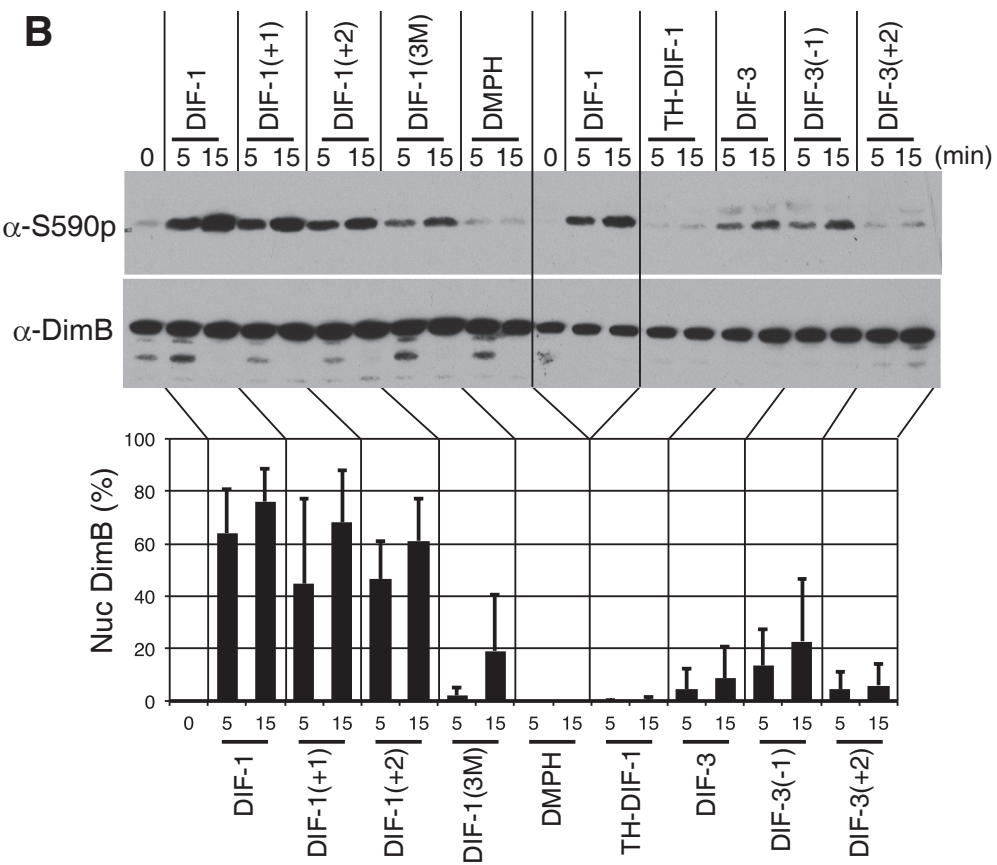

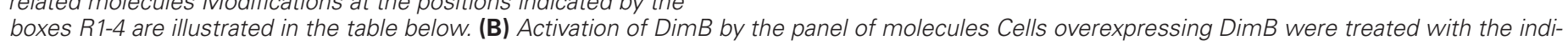

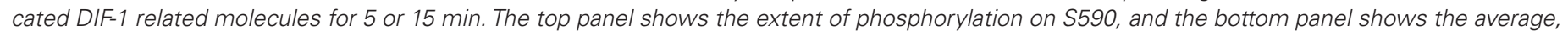
with SD, of the fraction of cells with nuclear-enriched DimB ( $n=6$ for DIF-1, $n=4$ for DIF-3, $n=3$ for the other compounds). 
A

Ax2

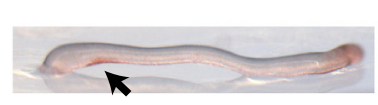

Neutral red
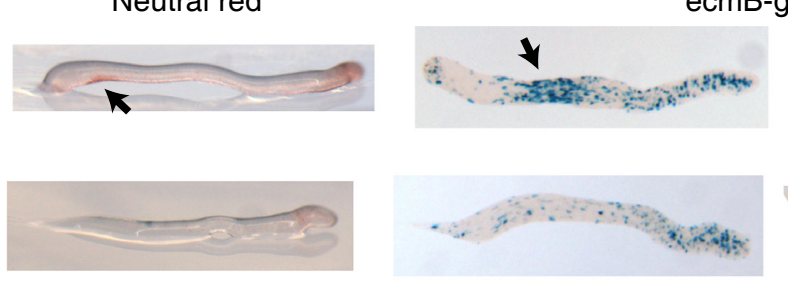

$\operatorname{dim} B-$

$\operatorname{dimB}$ - $/$

GFP-DimB:wt
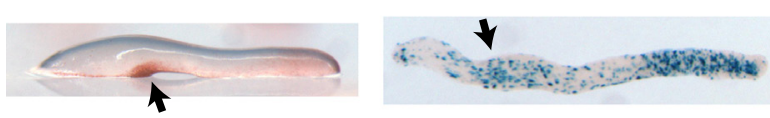

$\operatorname{dimB}$ - 1

GFP-DimB:S590A
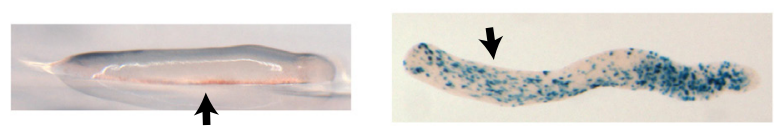

B

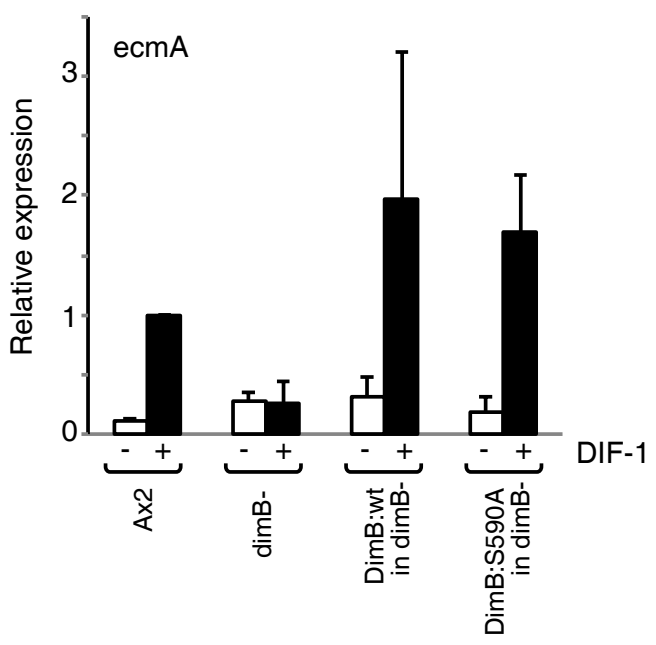

aspartic acid residues. Also, a triple mutant in peptide region $A$ was constructed, S3regA, that has all three phosphorylated serine residues changed into alanine. All of these constructs were transformed into DimB null cells and the nuclear translocation of the encoded DimB mutant proteins, in response to DIF-1, was compared with that of unmutated DimB. There is no statistically significant difference in the final extent of induction, which is essentially complete by 5 minutes (Fig. 3); however we cannot rule out a difference in kinetics of accumulation over the first 5 minutes because of technical limitations in the assay.

\section{Pharmacological evidence for a correlation between DimB nuclear accumulation and phosphorylation at S590}

Although we failed to detect an effect on nuclear translocation by mutating sites that change phosphorylation level in response to DIF-1, we are as stated probably only detecting a sub-set of such sites. There is the potential, therefore, for redundancy of function with other sites. Correlative evidence that this is likely to be true derives from analysis of a panel of molecules that share structural features with DIF-1.

The panel of compounds contains DIF-1 related molecules in which residues on the aromatic ring or the aliphatic side chain are altered (Fig. 4A). DMPH and TH-DIF-1 respectively lack the two chlorine atoms and the methoxy group of DIF-1. They are inactive as inducers of phosphorylation at S590 and as inducers nuclear accumulation of DimB.
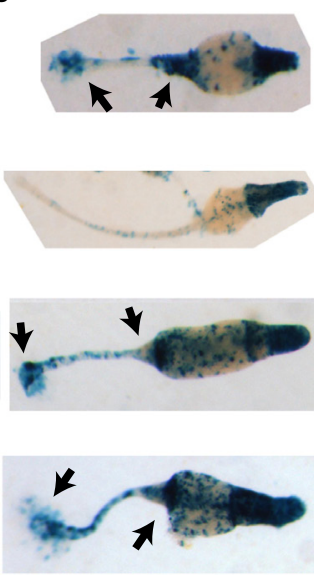

Fig. 5. Mutant rescue of developmental defects and DIF-1 inducible gene expression in the DimB null strain. (A) Rescue of developmental defects This figure shows the neutralred staining of migrating slugs (left) and in-situ enzymatic assays of ecmB-gal expression in migrating slugs (middle) and culminants (right) of parental, $\operatorname{dim} B$ - and $\operatorname{dim} B$ - cells expressing GFP-dimB in either the parental (WT) or the S590A mutant form. The pstB populations, in migrating slugs, and the lowercups and the basal discs of culminants are indicated with arrows. (B) Rescue of DIF-1 inducible gene expression This figure shows the induction of ecmA in parental Ax2 cells, dimB-cells and dimB-cells expressing DimB in either the parental (wt) or the S590A mutant forms.

of nuclear translocation (Fig. 4B). DIF-3 has one chlorine atom on the ring rather than two. It shows a low level of both phosphorylation and nuclear translocation. Increasing the length of the aliphatic side chain on DIF-3 by two carbon units inhibits both processes even further, while decreasing the chain length by one unit slightly increases both activities. Increasing the length of the side chain of DIF-1 itself, by one or two units, has only a small inhibitory effect but replacement of the terminal $n$ - butyl group with a tert-butyl group significantly reduces the induction of both phosphorylation and nuclear translocation. Thus there is a positive correlation between the efficacy of the various compounds as inducers of phosphorylation on S590 and as inducers of the

\section{The S590A mutant form of DimB reverts the phenotypes of a $\operatorname{dim} B$ null strain}

In order to determine whether the S590 site has a function other than regulating nuclear accumulation, as part of a transactivation domain for example, we performed mutant rescue analysis. The dimB- strain displays a typical DIF-1 signalling mutant phenotype; slugs split along their length very frequently, the spore head is often sub-apical and the fruiting bodies lack, or possess a much-reduced, basal disc and lower cup (Keller and Thompson, 2008; Thompson et al., 2004; Saito et al., 2008). The latter two structures derive from a group of cells that are ventrally located in the slug: the pstB cells. The pstB cells stain strongly with neutral red, express the emB-gal reporter highly and are much reduced or absent in dimB- slugs (Fig 5A). GFP-DimB:WT, is a construct with GFP fused to the $\mathrm{N}$ terminus of DimB, again under control of the DimB promoter but now cloned into a vector that confers blasticidin resistance. Such constructs are expected to be at single copy within the genome. When GFP-DimB:wt is transformed into dimB-cells the slug splitting and sub-apical spore head phenotypes are reverted (data not shown) and pstB cells and their derivative structures re-appear (Fig. 5A). The point-mutated DimB protein in an equivalent construct, GFP-DimB:S590A, is as effective as GFPDimB:wt in reverting the dimB- phenotypes. Also, the mutant form of DimB is effective in restoring DIF-regulated gene expression to $\operatorname{dimB}$ - cells in a monolayer assay; in parental cells the ecmA gene is inducible by DIF-1 but it is non DIF-inducible in dimB-cells 
A

100
DimB:wt NGDGSDDGSDTERENKKNRNRVNQNLASRNYRQRKKEYIKEIEEKLAVLALENDQLKKENINLKKGGGVEIMK

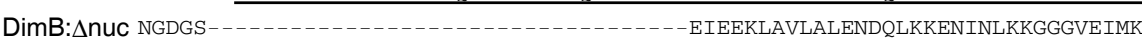

B

\section{C}

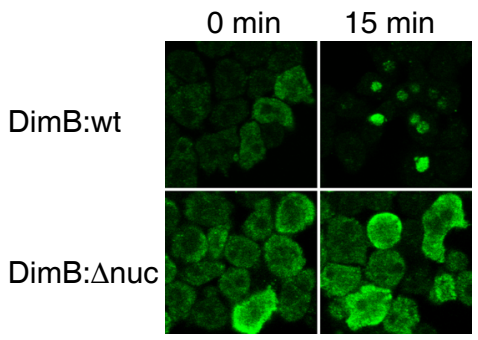

Fig. 6. Analysis of DIF-1 induced S590 phosphorylation in a DimB mutant protein defective in nuclear accumulation. (A) The structure of the mutant form This is the region of DimB containing the bZIP domain which is underlined, and a putative nuclear localization signal, highlighted in red. The sequence below indicates the deletion in DimB:Anuc used to prevent its nuclear accumulation. (B) Analysis of cellular localization of the parent and mutant forms DimB-derived cells expressing DimB:Anuc, were stimulated with DIF-1 and analysed for cellular localisation as in Fig. 3. (C) Analysis of S590 phosphorylation of the parent and mutant forms DimB-derived cells expressing DimB:wt or DimB:Anuc were stimulated with DIF-1 and analysed for 5590 phosphorylation as in Fig. 2. Note that the deleted, mutant protein runs more rapidly on the gel.
(Fig. 5B). In dimB- cells transformed, in this case with DimB:wt, ecmA inducibility is recovered. This is also true for $\operatorname{dimB}$ - cells transformed with DimB:S590A.

\section{The kinase activity that phosphorylates $\mathbf{S 5 9 0}$ is non-nuclear}

The kinase that phosphorylates DimB at S590 could be located outside the nucleus. There it could have a direct, but redundant, function in stimulating the nuclear import of DimB. Alternatively, the kinase could be nuclear localized, in which case it would only gain access to S590 after DimB had accumulated in the nucleus. In the latter case phosphorylation would be a consequence of translocation to the nucleus rather than a cause. In order to distinguish these possibilities we constructed DimB: $\Delta$ nuc; a mutant form of DimB wherein a predicted nuclear import signal-containing region is deleted (Fig. 6A). This was transformed into dimB- cells and immuno-fluoresence microscopy confirmed that the internally deleted DimB protein does, as expected, remain extra-nuclear after DIF-1 induction (Fig. 6B). Parallel Western transfer analysis shows that, despite being excluded from the nucleus, DimB: $\Delta$ nuc is inducibly phosphorylated on S590 (Fig. 6C). These observations suggest that DimB is phosphorylated, perhaps in the cytoplasm or at the plasma membrane, and then translocates to the nucleus.
The signaling pathway that directs phosphorylation of $\operatorname{dim} B$ differs significantly from the DIF-1 regulated pathway that activates STATC

We compared the DIF-induced DimB phosphorylation mechanism with that of STATc. There, Pyk2, a Tyrosine Kinase Like enzyme, phosphorylates STATc on a tyrosine residue, Y922, located close to the $\mathrm{C}$ terminus (Araki et al., 2012). This activation is constitutive and regulation by DIF-1 is exerted via DIF's inhibitory effect on PTP3; a protein tyrosine phosphatase that acts to dephosphorylate Y922 (Araki et al., 2008). DIF-1 inhibition of PTP3 correlates with the induced phosphorylation of two PTP3 serine residues: S448 and S747. These two serine modifications, and the activation of STATc itself, are stimulated by BHQ; a reagent that elevates intracellular calcium levels (Araki et al., 2010). While BHQ is, as expected, an inducer of the tyrosine phosphorylation and nuclear accumulation of STATc, it does not stimulate S590 phosphorylation or nuclear accumulation of DimB (Fig 7A). Furthermore, in a pyk2 null strain STATc is, as expected, unresponsive to DIF-1 but DimB remains responsive for both $\mathrm{S} 590$ phosphorylation and nuclear accumulation (Fig 7B). Thus the two pathways do not overlap at the levels analysed here.
A
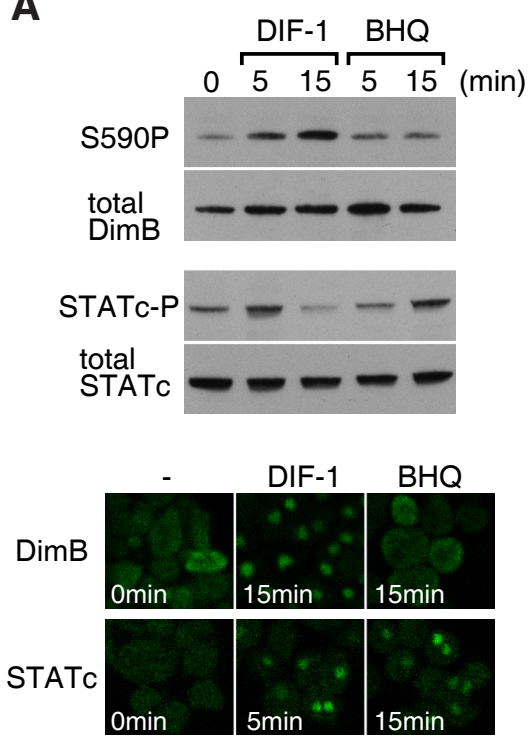

DimB

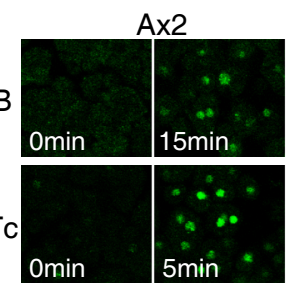

B

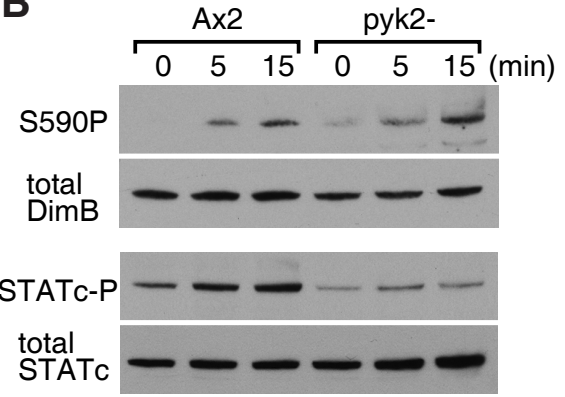

Fig. 7. A comparison of DimB and STATc activation. (A) The effect of $B H Q$ stimulation Ax2 derived cells, over-expressing DimB, were incubated with 100nM DIF-1 or 30u M BHQ for the indicated times. Phosphorylation at S590 and nuclear translocation of DimB were determined as in Fig. 2 and Fig. 3 respectively. (B) The effect of genetically ablating Pyk2 Parental Ax2 and pyk2-cells were induced with 100nM DIF-1 and phosphorylation at S590 and nuclear translocation of DimB were determined as in Fig. 2 and Fig. 3 respectively. 


\section{Discussion}

Upon stimulation of cells with DIF-1 there is an increase of phosphorylation in two regions of DimB; a small increase in region $A$, that is located in the bZIP domain, and a much larger increase in a C terminus-proximal region: $B$. The identification of potential sites of phosphorylation in region $A$ is based purely upon mass spectrometry. However, a change in phosphorylation at one of the three predicted sites of region B, S590, was confirmed using a phospho-specific antibody. The S590 flanking sequence is proline-rich. It is perhaps, therefore, a substrate for a proline-directed kinase but we do not know its identity.

The function of S590 was investigated by monitoring nuclear accumulation of a non-phosphorylatable $S$ to $A$ form. The mutant form accumulates in the nucleus normally after DIF-1 induction. Although we were unable to discern an effect of the mutation on nuclear accumulation we believe that this is likely to result from redundancy in the signalling pathway that directs the process. Part of the reason for believing that there is another, redundant, site derives from analysis of a panel of DIF-1 related molecules. There is a good correlation between the ability of a compound to direct DimB phosphorylation at S590 and its ability to induce nuclear accumulation. It is perhaps relevant to compare the present data on activation of DimB with published results on the potency of the same panel as inducers of stalk cell differentiation in the DIF-deficient HM44 mutant (Gokan et al., 2005). There is generally a reasonable correlation, the one notable exception being TH-DIF-1; a good inducer of stalk cells that is relatively inactive in inducing $\mathbf{S} 590$ phosphorylation and nuclear translocation of DimB. This probably reflects the fact that TH-DIF-1 is a biosynthetic precursor of DIF-1 and, over the relatively long incubation period of the stalk cell assay, sufficient DIF-1 is produced to induce stalk cell formation. A further, definitive demonstration that the S590 site is non-essential is the ability of the S590A mutant form to revert the various phenotypes of the $\operatorname{dimB}$ null strain.

Although we cannotassign a definitive function to the phosphorylation of S590, the phospho-specific antibody is a tool for investigating the cognate, DIF-regulated signalling pathway; the S590 antibody makes possible a biochemical assay for the kinase and therefore a route to its purification. One very relevant issue in assessing its usefulness in this respect is the intracellular site of phosphorylation. If this were nuclear rather than extra-nuclear, such that phosphorylation was dependent upon accumulation in the nucleus rather than being directly responsive to DIF, this would negate its usefulness. However, analysis of a partial deletion mutant of DimB that does not accumulate in the nucleus supports a direct response to DIF-1.

Finally, we used the antibody to determine whether there is an overlap with the DIF-regulated STATc activation pathway. This pathway has two arms, a constitutive phosphorylation arm and a DIF-regulated de-phosphorylation pathway. Genetic ablation of the kinase arm and pharmacological stimulation of the phosphatase arm were both without effect on S590 phosphorylation of DimB. This implies that the STATC and DimB pathways are separate at the levels analysed but leaves open the possibility that they share upstream steps.

\section{Materials and Methods}

Cell culture, development and DIF-1 induction

Dictyostelium strain Ax2 (Gerisch isolate) cells were grown axenically at $21^{\circ} \mathrm{C}$ in $\mathrm{HL}-5$ medium. For induction in suspension, the cells were starved for four hours in KK2 buffer (16.5 mM KH $\mathrm{PO}_{4}, 3.8 \mathrm{mM} \mathrm{K}_{2} \mathrm{HPO}_{4}, \mathrm{pH}$ 6.2) at a concentration of $2 \times 10^{7} \mathrm{cells} / \mathrm{ml}$, by shaking at $200 \mathrm{rpm}$. DIF-1 (Enzo Life Sciences (UK) Ltd, Exeter, UK) was then added to $100 \mathrm{nM}$ and shaking was continued. Western transfer analysis and immuno-chemical staining of the induced cells were performed as described previously (Araki et al., 2003), using either a general DimB antibody or the newly created phosphospecific S590 antibody. For mass spectrometry analysis of phosphorylation sites, cells were developed on water agar plates until the loose mound stage. The mounds were then dissociated in KK2 buffer and DIF-1 was added to $100 \mathrm{nM}$, before shaking as above. DIF-related compounds were synthesized as described previously (Gokan et al., 2005).

\section{Parent ion scanning mass spectrometry for the identification of phosphorylation sites}

Ax2 cells over-expressing DimB were left untreated or treated with DIF-1 for $15 \mathrm{~min}$. They were then lysed and fractionated, by immuno-precipitation followed by SDS gel electrophoresis. After purification, samples were separated by SDS-PAGE. The selected bands were digested, normally with chymotrypsin, and injected onto a nLC-MS-MS analysis system. UV chromatography showed that the amounts of protein loaded onto the LC system for each sample were comparable. The samples were subjected to parent-ion scanning, searching for peptides that generate a phosphate ion. The relative amount of phosphopeptides in control and DIF-1 treated samples was quantitated using Analyst (AB Sciex, USA) software. Sequences of the parent ions and sites of phosphorylation were then deduced by MS to MS analysis. In some cases a list of the masses of phosphopeptides was made from the pre79 results and inclusion list, nLC-MS-MS analysis was performed. The coverage of the protein was estimated to be $30 \%$ for chymotrypsin. In separate experiments trypsin, either with or without AspN, was used for digestion and then somewhat over $50 \%$ of residues were accessible. The overlay patterns were complex; because of different protease mis-cleavage products, different oxidation states, different bound small ions or different numbers of phosphate groups on peptides derived from the same protein region. Also, some of the peaks that appeared to coincide on the overlaid scans derived from peptides that eluted at different times in the liquid chromatography step; they were therefore deemed false positives.

\section{Generation of phospho-specific antibodies and immuno-chemical techniques}

Phospho-specific DimB antibodies were raised by injecting KLH conjugated phosphorylated peptides into sheep and was purified, as described, using sequential phospho-peptide and non-phosphopeptide column chromatography (Araki et al., 2008).

\section{Acknowledgements}

This work was supported by Wellcome Trust Program Grant 082579/Z (JGW) and Grants-in-aid for Scientific Research from the Ministry of Education, Culture, Sports, Science and Technology of Japan (YK, YO, HK).

\section{References}

ARAKIT, KAWATAT, and WILLIAMS J G (2012) Identification of the kinase that activates a nonmetazoan STAT gives insights into the evolution of phosphotyrosine-SH2 domain signaling. Proc Natl Acad Sci USA 109: 1931-1937

ARAKI T, LANGENICK J, GAMPER M, FIRTEL, R A and WILLIAMS J G (2008) Evidence that DIF-1 and hyper-osmotic stress activate a Dictyostelium STAT by inhibiting a specific protein tyrosine phosphatase. Development. 135: 1347-1353.

ARAKI T, TSUJIOKA M, ABE T, FUKUZAWA M, MEIMA M, SCHAAP P, MORIO T, URUSHIHARAH, KATOH M, MAEDAM, TANAKAY, TAKEUCHII and WILLIAMS, J.G. (2003) A STAT-regulated, stress-induced signalling pathway in Dictyostelium. J. Cell Sci, 116: 2907-2915.

ARAKI, T, VAN EGMOND W N, VAN HAASTERT P J, AND WILLIAMS J G (2010) Dual regulation of a Dictyostelium STAT by cGMP and $\mathrm{Ca2}+$ signalling. $J$ Cell 
Sci: 123, 837-841.

EARLY A E, GASKELL M J, TRAYNOR D, AND WILLIAMS J G (1993) Two distinct populations of prestalk cells within the tip of the migratory Dictyostelium slug with differing fates at culmination. Development 118: 353-362.

GAUDETP, WILLIAMS J G, FEYP and CHISHOLM RL (2008)An anatomy ontology to represent biological knowledge in Dictyostelium discoideum. BMC Genomics 9: 130.

GOKAN N, KIKUCHI H, NAKAMURA K, OSHIMA Y, HOSAKAK and KUBOHARA, Y. (2005) Structural requirements of Dictyostelium differentiation-inducing factors for their stalk-cell-inducing activity in Dictyostelium cells and anti-proliferative activity in K562 human leukemic cells. Biochem. Pharmacol. 70: 676-685.

HUANG E, BLAGG S L, KELLER T, KATOH M, SHAULSKY G and THOMPSON C $\mathrm{R}$ (2006) bZIP transcription factor interactions regulate DIF responses in Dictyostelium. Development 133: 449-458.

JERMYN K A, TRAYNOR D and WILLIAMS J G (1996) The initiation of basal disc formation in Dictyostelium discoideum is an early event in culmination. Development 122: 753-760.

KAY R R and THOMPSON C R (2009) Forming patterns in development without morphogen gradients: scattered differentiation and sorting out. Cold Spring Harb Perspect Biol 1: a001503

KAY R R and THOMPSON C R L (2001) Cross-induction of cell types in Dictyostelium: evidence that DIF-1 is made by prespore cells. Development 128: 4959-4966.
KELLER T and THOMPSON C R (2008) Cell type specificity of a diffusible inducer is determined by a GATA family transcription factor. Development 135: 1635-1645.

SAITO T, KATO A. and KAY R R (2008) DIF-1 induces the basal disc of the Dictyostelium fruiting body. Dev Biol. 317: 444-453.

SHARMA N, LOPEZ D I and NYBORK J K (2007) DNA binding and phosphorylation induce conformational alterations in the kinase-inducible domain of CREB. Implications for the mechanism of transcription function. J Biol Chem 282: 19872-19883.

THOMPSON C R L and KAY R R (2000) The role of DIF-1 signaling in Dictyostelium development. Mol. Cell 6: 1509-1514.

THOMPSON C R L, FU Q, BUHAY C, KAY R R and SHAULSKY G. (2004) A bZIPI $\mathrm{bRLZ}$ transcription factor required for DIF signaling in Dictyostelium. Development 131: 513-523.

TSUKADA J, YOSHIDA Y, KOMINATO Y, and AURON P E (2011) The CCAAT/ enhancer (C/EBP) family of basic-leucine zipper (bZIP) transcription factors is a multifaceted highly-regulated system for gene regulation. Cytokine 54: 6-19.

YAMADA Y, NUNEZ-CORCUERA B. and WILLIAMS J G (2011). DIF-1 regulates Dictyostelium basal disc differentiation by inducing the nuclear accumulation of a bZIP transcription factor. Dev Biol 354: 77-86.

ZHUKOVSKAYAN V, FUKUZAWAM, YAMADAY,ARAKITAND WILLIAMS J G (2006) The Dictyostelium bZIP transcription factor DimB regulates prestalk-specific gene expression. Development 133: 439-448. 


\section{Further Related Reading, published previously in the Int. J. Dev. Biol.}

An orthologue of the Myelin-gene Regulatory Transcription Factor regulates Dictyostelium prestalk differentiation Hiroshi Senoo, Hong-Yu Wang, Tsuyoshi Araki, Jeffrey G. Williams and Masashi Fukuzawa Int. J. Dev. Biol. (2012) 56: 325-334

Bimodal distribution of motility and cell fate in Dictyostelium discoideum Pavana Goury-Sistla, Vidyanand Nanjundiah and Gopal Pande Int. J. Dev. Biol. (2012) 56: 263-272

A SET/MYND chromatin re-modelling protein regulates Dictyostelium prespore patterning Beatriz Nuñez-Corcuera, Joanna Birch and Jeffrey G. Williams Int. J. Dev. Biol. (2011) 55: 205-208

Analysis of a homologue of the adducin head gene which is a potential target for the Dictyostelium STAT protein Dd-STATa Ryota Aoshima, Rieko Hiraoka, Nao Shimada and Takefumi Kawata Int. J. Dev. Biol. (2006) 50: 523-532

Identification of new modes of Dd-STATa regulation of gene expression in Dictyostelium by in situ hybridisation Nao Shimada, Mineko Maeda, Hideko Urushiharaand Takefumi Kawata

Int. J. Dev. Biol. (2004) 48: 679-682

The MADS-box transcription factor SRFA regulates different aspects of Dictyostelium discoideum development

N Moreno, JJ Vicente, R Escalante, L Sastre

Int. J. Dev. Biol. (2001) 45: S117-S118

Cell cycle phase, cellular Ca2+ and development in Dictyostelium discoideum MAzhar, PK Kennady, G Pande, M Espiritu, W Holloman, D Brazill, RH Gomer and V Nanjundiah Int. J. Dev. Biol. (2001) 45: 405-414

The MADS-box transcription factor SRFA regulates different aspects of Dictyostelium discoideum development

N Moreno, JJ Vicente, R Escalante, L Sastre

Int. J. Dev. Biol. (2001) 45: S117-S118

A cell type-specific effect of calcium on pattern formation and differentiation in dictyostelium discoideum

R Baskar, P Chhabra, P Mascarenhas and V Nanjundiah

Int. J. Dev. Biol. (2000) 44: 491-498

5 yr ISI Impact Factor $(2011)=2.959$

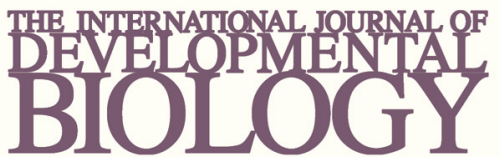

Volume 54 Nos. $6 / 7$
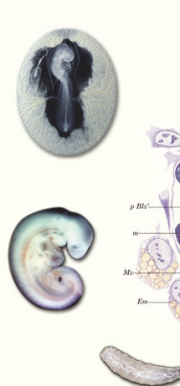

Developmental Hematopoiesis
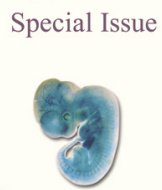

(2) (8)
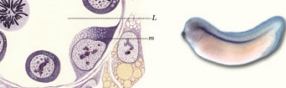

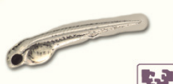

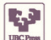

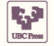

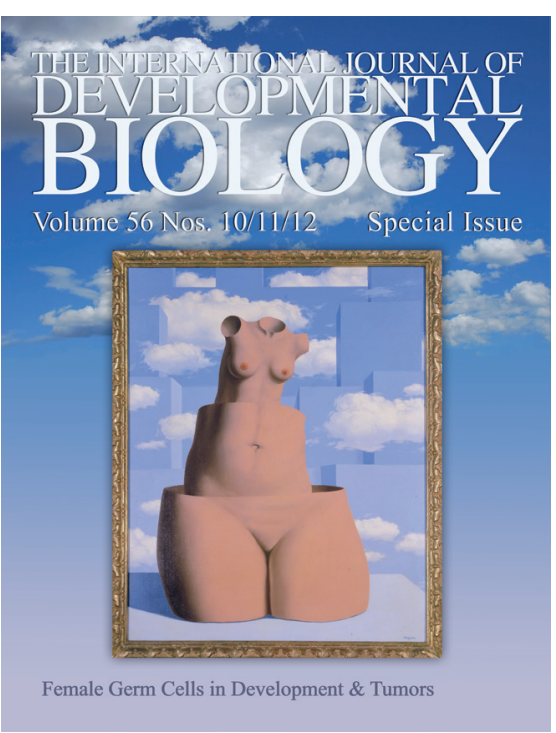
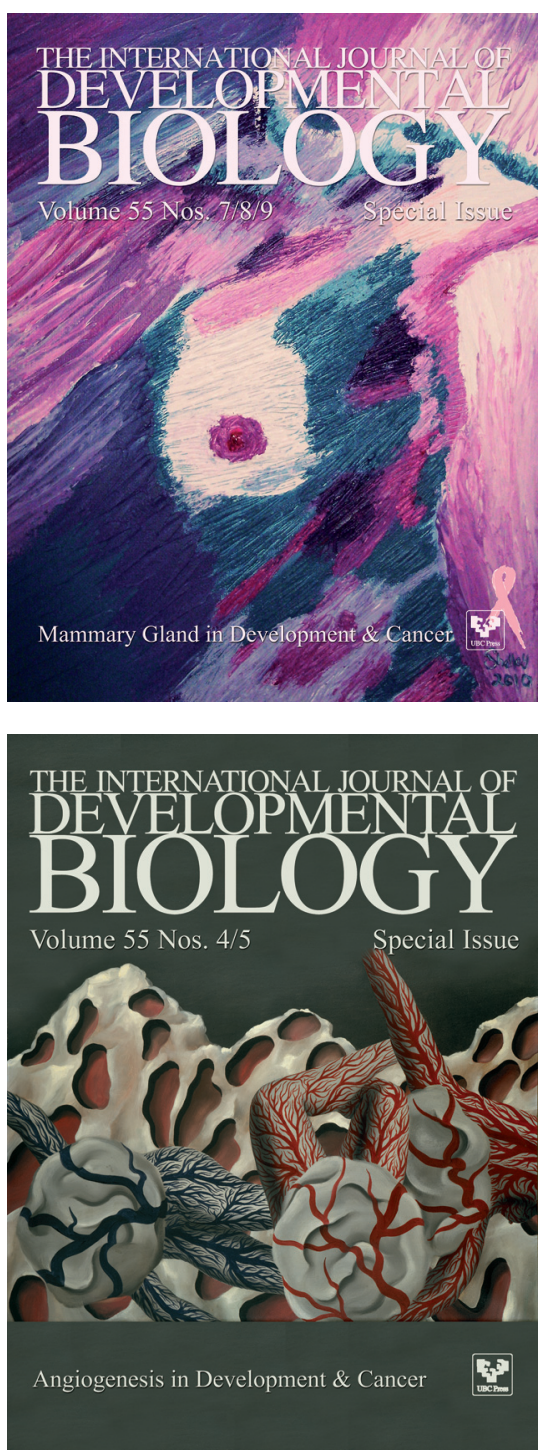